Document downloaded from:

http://hdl.handle.net/10251/74058

This paper must be cited as:

Payri González, F.; Broatch Jacobi, JA.; Serrano Cruz, JR.; Piqueras Cabrera, P. (2011). Experimental theoretical methodology for determination of inertial pressure drop distribution and pore structure properties in wall-flow diesel particulate filters (DPFs). Energy. 36(12):6731-6744. doi:10.1016/j.energy.2011.10.033.

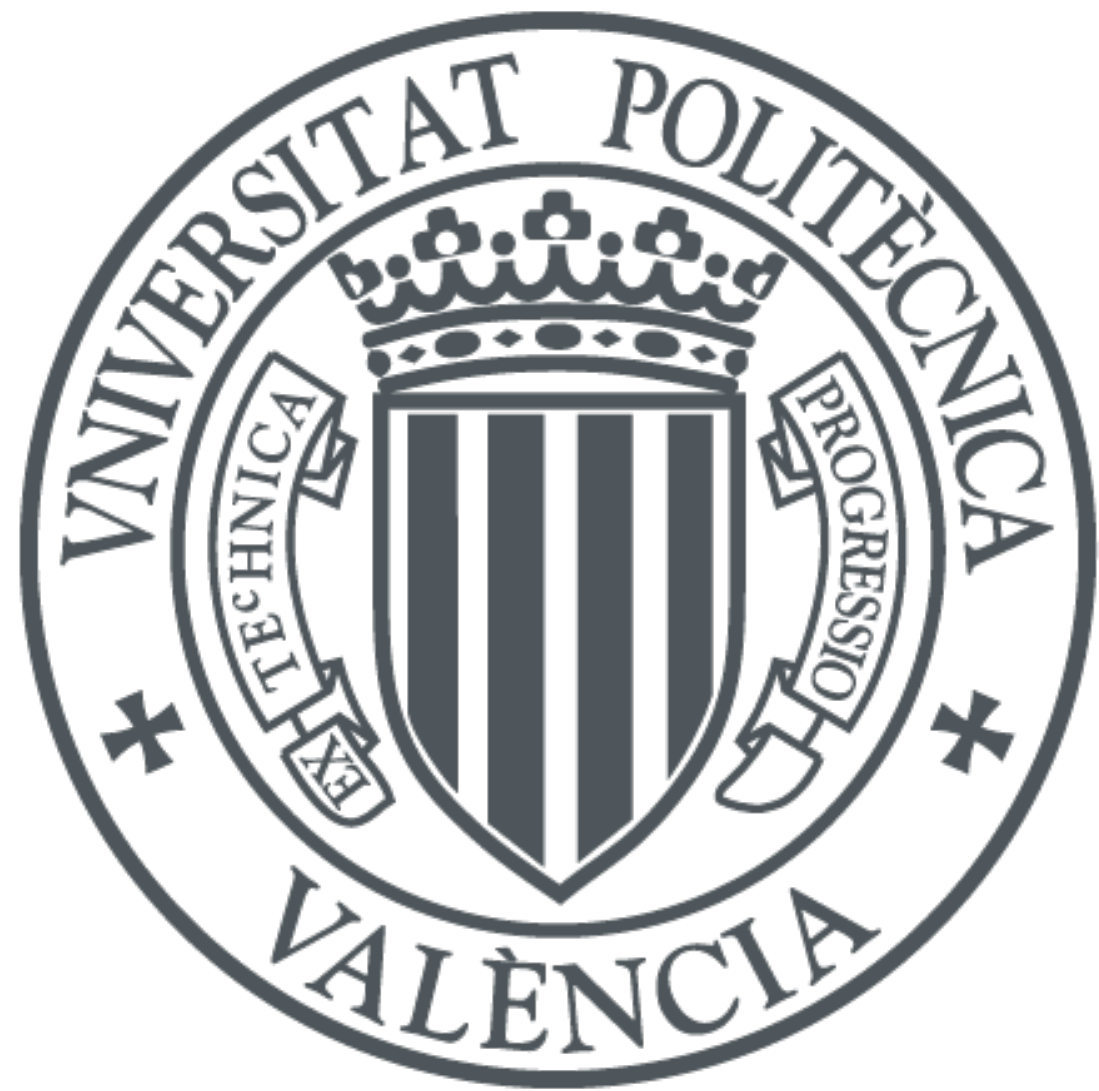

The final publication is available at

http://dx.doi.org/:10.1016/j.energy.2011.10.033

Copyright Elsevier

Additional Information 


\title{
Experimental-theoretical methodology for determination of inertial pressure drop distribution and pore structure properties in wall-flow diesel particulate filters
}

\author{
F. Payri, A. Broatch, J. R. Serrano, P. Piqueras* \\ Universitat Politècnica de València, CMT-Motores Térmicos, Camino de Vera s/n, 46022 Valencia, Spain.
}

\begin{abstract}
Wall-flow particulate filters have been placed as a standard technology for Diesel engines because of the increasing restrictions to soot emissions. The inclusion of this system within the exhaust line requires the development of computational tools to properly simulate its flow dynamics and acoustics behaviour. These aspects become the key to understand the influence on engine performance and driveability as a function of the filter placement. Since the pressure drop and the filtration process are strongly depending on the pore structure properties - permeability, porosity and pore size - a reliable definition of these characteristics is essential for model development. In this work a methodology is proposed to determine such properties based on the combination of the pressure drop rement in a steady flow test rig and two theoretical approaches. The later are a lumped model and a one-dimensional unsteady compressible flow model. The purpose is to simplify the integration of particulate filters into the global engine modelling and development processes avoiding the need to resort to specific and expensive characterisation tests. The proposed methodology was validated against measurements of the response of an uncoated DPF under different flow conditions as cold steady flow, impulsive flow and hot pulsating flow.
\end{abstract}

Keywords: Diesel particulate filter, porous media, permeability, slip-flow, gas dynamics, experiments

\section{Introduction}

Diesel particulate filters (DPF) appear to be the most reliable approach to tackle soot particulate emissions in Diesel engines and hence its study for almost thirty years ago. Nevertheless, its widespread use has not been reached up to just ten years ago [1]. The reasons arise from several interdisciplinary aspects. For many years, the limits to soot and the remainder emissions imposed by the pollutant regulations have been overcome by means of improvements on the injection and the combustion processes and technology [2,3], the comprehensive understanding of pollutants formation during transient behaviour of Diesel engines [4, 5], flexible turbocharging systems [6] or exhaust gas recirculation control [7]. The solutions proposed in these research fields have delayed the introduction of the DPF, whose early use would have meant to deal with important problems regarding safe, reliable and periodic regeneration under

${ }^{*}$ P. Piqueras. CMT-Motores Térmicos, Universitat Politècnica de València, Camino de Vera s/n, 46022 Valencia, Spain. Phone: +34 963877650 Fax: +34 963877659 e-mail: pedpicab@mot.upv.es 
all driving conditions. Additionally, available regeneration techniques to drive the system towards low back pressure, implying minimum fuel consumption, were complex and costly.

However, increasingly restrictive regulations $[8,9]$ have led manufacturers and researchers to resort to DPF systems in an immediate future. These systems guarantees a proven filtration efficiency to reduce all the particulate emission range size, from $10 \mathrm{~nm}$ up to two or three order of magnitude over [10]. This context has motivated sound improvements in soot filtration technology in recent years [11] on the basis of the first studies covering the keys for a comprehensive understanding of the DPF operation [12].

The development of DPFs has comprised the exploration of different systems defined by the combination of materials and flow path-lines: ceramic honeycomb wall-flow monoliths, ceramic fibres, ceramic foams, metal fibre filters or sintered metals. Among these solutions, honeycomb wall-flow monoliths, which are mainly made of cordierite or silicon carbide, have achieved the most widespread commercial implantation covering both passenger and heavy-duty transport [13]. This type of monolithic filters consists of a bundle of small axial parallel channels, typically of square cross section. Adjacent channels are alternatively plugged at each end in order to force the gas flow through the porous substrate wall from the inlet to the outlet channels. The soot particulates are filtrated and accumulated into or on the porous wall until regeneration. The main advantages of this sort of filters is a good balance in properties regarding high filtration efficiency, specific surface area and thermal shock resistance combined with flexibility to diversity of active and passive regeneration strategies.

The parameters defining the geometry of DPF monoliths quoted by the filter manufacturers are nominal values. However, raw material and extrusion process variability or material changes during the thermal treatment of the ceramic material are all factors that may affect the exact values of these parameters [14]. To overcome this problem is specially important in the case of the pore structure properties because of their influence on the characterisation of pressure drop [15], filtration [16] and regeneration [17].

The evaluation of the clean permeability of the porous substrate in wall-flow DPFs has been proposed in the literature through the testing of the isolated monolith [14]. As drawback, this methodology requires an independent characterisation of the inertial pressure drop in the inlet and outlet cones of the canned DPF to properly study the influence of the DPF on engine performance [18].

In this work a characterisation methodology to determine both the permeability in clean wall-flow monoliths and all the inertial pressure drop contributions in a canned DPF is proposed exploring the relation between experimental and modelling techniques. It also allows the description of the pore structure characteristics of the substrate, which is needed to account for the slip-flow effect on the DPF pressure drop at high temperature.

The proposed methodology is based on the direct testing of a canned DPF system under cold steady flow, which provides the pressure drop produced by the whole device. This test avoids dismantling the DPF to only test the monolith and the uncertainty of the best place where pressure sensors should be located [19] is overcome. The measured pressure drop is the addition of those produced by the inlet and outlet ducts to the filter and by the different 
flow interactions taking place at the different components: inlet and outlet volumes, monolith channels and porous medium.

The coexistence of several non-inertial and inertial contributions to the pressure drop, which influence each other, prevents from a direct analytical determination of the porous wall permeability. As a consequence, experimental data are used as control parameter of an iterative computation procedure. It drives both the determination of the permeability and the decomposition of every of the additional non-inertial and inertial mechanisms inducing the pressure drop. The computation couples a lumped parameter model for pressure drop prediction across wall-flow DPFs and a one-dimensional (1D) model solving the unsteady compressible flow equations in DPF channels. The porosity and the mean pore size are assessed from the permeability value and the theoretical background about packed beds of spherical particles. The obtained definition of the pressure drop distribution and the pore structure is experimentally validated applying it to the prediction of the DPF flow dynamics response under cold steady flow, impulsive flow and hot pulsating flow.

\section{Determination of permeability and inertial pressure drop distribution}

The proposed methodology quantifies the permeability of the clean porous substrate in honeycomb wall-flow DPFs and characterises the contribution to the pressure drop of the rest of singularities. The required experimental information is the pressure drop produced by the filter. It can be also assessed with modelling tools, so that the contributions of both inertial and non-inertial mechanisms can be evaluated.

\subsection{Lumped parameter pressure drop model for wall-flow DPFs}

Konstandopoulos [14] proposed a lumped parameter model for the pressure drop calculation in honeycomb wallflow monoliths based on an incompressible flow approach. The pressure drop of an isolated clean monolith is given by:

$$
\Delta p_{\text {mon }}=\frac{\mu Q}{2 V_{\text {mon }}}\left(\alpha+w_{w}\right)^{2}\left(\frac{w_{w}}{k_{w} \alpha}+\frac{8 F_{w} L^{2}}{3 \alpha^{4}}\right)+\frac{\rho Q^{2}}{V_{m o n}^{2} \alpha^{2}}\left(\alpha+w_{w}\right)^{4}\left(\frac{\beta_{F} w_{w}}{4}+2 \varsigma_{\text {mon }}\left(\frac{L}{\alpha}\right)^{2}\right)
$$

On the one hand, the non-inertial mechanisms are correlated with the volumetric flow rate $(Q)$ and controlled by the porous wall permeability $\left(k_{w}\right)$ and the friction process. The pressure drop due to friction in square porous channels depends on the momentum transfer coefficient $F_{w}$, whose value is constant and equal to 28.454 [12]. On the other hand, the inertial terms, which are proportional to the squared volumetric flow rate, include the following contributions:

- Pressure drop across the porous wall by means of the Forchheimer's coefficient $\left(\beta_{F}\right)$.

- Pressure drop due to the local contraction of the flow entering to the inlet channels.

- Pressure drop due to the local expansion of the flow coming out of the outlet channels. 
The last two contributions are considered together in a solely pressure drop coefficient $\left(\varsigma_{\text {mon }}\right)$ in equation (1).

In order to extent the proposal of Konstandopoulos to calculate the pressure drop of the canned DPF device, i.e. accounting for the pressure drop coefficients related to the expansion at the inlet cone of the filter $\left(\varsigma_{i e}\right)$ and the contraction at the outlet cone $\left(\varsigma_{o c}\right)$, equation $(1)$ can be rewritten as:

$$
\Delta p_{D P F}=\frac{\mu Q}{2 V_{m o n}}\left(\alpha+w_{w}\right)^{2}\left(\frac{w_{w}}{k_{w} \alpha}+\frac{8 F_{w} L^{2}}{3 \alpha^{4}}\right)+\frac{\rho Q^{2}}{V_{m o n}^{2} \alpha^{2}}\left(\alpha+w_{w}\right)^{4}\left(\frac{\beta_{F} w_{w}}{4}+2\left(\varsigma_{m o n}+\varsigma_{i e}+\varsigma_{o c}\right)\left(\frac{L}{\alpha}\right)^{2}\right)
$$

The ratio between the pressure drop and the volumetric flow rate can be expressed by a linear function of the volumetric flow rate as,

$$
\frac{\Delta p_{D P F}}{Q}=a+b Q
$$

where coefficients $a$ and $b$ are respectively:

$$
\begin{gathered}
a=\frac{\mu}{2 V_{m o n}}\left(\alpha+w_{w}\right)^{2}\left(\frac{w_{w}}{k_{w} \alpha}+\frac{8 F_{w} L^{2}}{3 \alpha^{4}}\right) \\
b=\frac{\rho}{V_{m o n}^{2} \alpha^{2}}\left(\alpha+w_{w}\right)^{4}\left(\frac{\beta_{F} w_{w}}{4}+2\left(\varsigma_{m o n}+\varsigma_{i e}+\varsigma_{o c}\right)\left(\frac{L}{\alpha}\right)^{2}\right)
\end{gathered}
$$

Experimental data provide the pressure drop and the volumetric flow rate to obtain the coefficient $a$. As a consequence, knowing the coefficient $a$ and the geometry of the DPF, the permeability of the porous wall can be determined by means of equation (6):

$$
k_{w}=\frac{w_{w}}{\alpha}\left(\frac{2 V_{\text {mon }} a}{\mu\left(\alpha+w_{w}\right)^{2}}-\frac{8 F_{w} L^{2}}{3 \alpha^{4}}\right)^{-1}
$$

\subsection{Experimental procedure and data processing}

In this work, a standard uncoated DPF for a 21 Diesel engine was tested in a steady flow test rig at room temperature. Table 1 shows the geometrical data of the tested DPF.

Tests with mass flow rates ranging between $80 \mathrm{~kg} / \mathrm{h}$ and $400 \mathrm{~kg} / \mathrm{h}$ were performed in order to measure the pressure drop across the DPF at a wide range of flow regimes, with Reynolds number (Re) from 30000 to 150000 at the inlet of the DPF.

Figure 1 shows a scheme of the set-up used to test the DPF. Tests were performed in aspiration inducing a pressure decrease in the settling volume by means of a roots blower. The air mass flow rate was measured with a hot film anemometer whereas the pressure drop was measured with a water column connected to the settling volume. With this set-up, the measured pressure drop includes the contribution of the inlet duct, the canned DPF, the outlet duct and the kinetic energy loss of the flow discharged into the settling volume. This can be expressed mathematically as

$$
\Delta p_{D P F} \text { test }=\Delta p_{i d}+\Delta p_{D P F}+\Delta p_{o d}+\Delta p_{o d_{s v}}
$$


where $\Delta p$ represents the stagnation pressure drop; subscript $i d$ refers to the pressure drop at the inlet duct, which includes the entrance and friction losses; subscript $o d$ is referred to the pressure drop at the outlet duct due to friction; subscript $s v$ indicates the pressure drop due to the discharge into the settling volume.

To determine the porous wall permeability accurately, the contributions of all singularities to pressure drop must be quantified. With this purpose, the inlet duct was tested independently, so that its particular pressure drop was characterised. Following the same procedure that in the case of the filter, the pressure drop obtained from this test is:

$$
\Delta p_{i d \text { test }}=\Delta p_{i d}+\Delta p_{i d_{s v}}
$$

Therefore, rearranging equation (7) and combining with equation (8), the pressure drop of the canned DPF plus that due to friction in the outlet duct can be determined empirically as:

$$
\Delta p_{D P F}+\Delta p_{o d}=\Delta p_{D P F} \text { test }-\Delta p_{o d_{s v}}-\Delta p_{i d \text { test }}+\Delta p_{i d_{s v}}
$$

Since the dynamic pressure of the flow discharged into the settling volume is lost, the pressure drop produced by such singularities is

$$
\Delta p_{o d_{s v}}=\frac{1}{2} \frac{\dot{m}^{2}}{\rho F_{o d}^{2}} \quad \Delta p_{i d_{s v}}=\frac{1}{2} \frac{\dot{m}^{2}}{\rho F_{i d}^{2}}
$$

being $\rho$ the air density at room conditions because of incompressible flow assumption.

According to this procedure, experiments in the steady flow test rig yield the information shown in Figure 2. The pressure drop induced by the DPF cannot be isolated experimentally from that caused by the friction in the outlet duct. Therefore, the complete decomposition of the pressure drop contributions cannot be achieved only with an experimental approach.

As a consequence, the solely application of the lumped parameter model (equation (6)) to obtain the permeability is not possible. To surpass this limitation a one-dimensional modelling of the experiments is proposed to complete the characterisation. The use of a one-dimensional gas dynamics code can provide the friction pressure drop across the outlet duct of the installation and the complete decomposition of the inertial pressure drop across the canned DPF.

\subsection{One-dimensional model for wall-flow DPFs}

The 1D model for honeycomb wall-flow DPFs includes the solution of the flow unsteadiness, characteristic of internal combustion engines dynamics. The main aspects of the model formulation are described in Appendix A. Further description of the model can be found in detail in [20, 21].

The solution of the flow dynamics inside the monolith assumes that the flow is uniform at the inlet cross-section of the monolith (but time-dependant) and that the monolith is perfectly insulated in the radial direction under cold flow operation. Therefore, all the pairs of inlet-outlet channels have the same behaviour and only the solution of one of them is required [12]. The solution of the monolith channels allows to evaluate the pressure drop due to non-inertial 
mechanisms, i.e. flow through the porous medium and flow-wall friction. The remaining pressure drop contributions are considered by means of the modelling of the inlet and outlet volumes of the DPF.

The DPF model has been implemented in OpenWAM ${ }^{\mathrm{TM}}$ (Open Wave Action Model) [22], an open source gas dynamics software which has been developed at CMT-Motores Térmicos. The DPF model can be coupled to the inlet and the outlet ducts of the DPF for the simulation of either test facilities for characterisation purpose or real engine operation.

The large cone angle of the inlet and outlet volumes of the DPF does not permit a one-dimensional modelling of these elements [23]. The simplest and most suitable solution to this limitation is to simplify the problem simulating both volumes of the DPF as OD elements. Figure 3 shows the representation of the DPF in the OpenWAM's interface. In this figure, the big rectangles represent $0 \mathrm{D}$ elements and the medium size squares, numbered from 1 to 6 , represent boundary conditions. The 1D elements, such as ducts and monolith channels, are represented by solid lines connecting the boundary conditions. The monolith channel numbered as 1 represents an inlet channel whereas the channel 2 is the outlet channel. The grey arrows indicate the flow through the porous medium, which is considered as source term in the conservation equations.

The inertial pressure drop contributions are modelled by boundary conditions connecting 0D and 1D elements. These boundaries, based on the Benson's proposal [24], calculate the pressure drop at the inlet and outlet of every volume defining discharge coefficients. According to the numbering shown in Figure 3, the following boundaries have been used:

- Boundary condition 1 accounts for the pressure drop due to the flow expansion at the inlet cone of the filter.

- Boundary condition 2 accounts for the pressure drop due to the local flow contraction when entering to the inlet monolith channels.

- Boundary condition 5 accounts for the pressure drop due to the local flow expansion when exiting from the outlet monolith channels.

- Boundary condition 6 accounts for the pressure drop due to the flow contraction in the outlet cone of the filter.

The monolith description is completed with the inclusion of the boundary conditions 3 and 4, which represent the alternatively plugged ends at inlet and outlet channels.

\subsection{Calculation iterative procedure}

The proposed procedure for the characterisation of both the permeability and the remaining contributions to the pressure drop in clean honeycomb wall-flow DPFs is sketched in Figure 4.

The first phase concerns the testing of the canned DPF and the inlet duct in the steady flow test rig according to the description given in section 2.2. The pressure drop across the canned DPF and the outlet duct can be determined by means of the post-processing of the experimental data, as shown in Figure 2. From these data, the modelling of the 
experiments coupling the lumped parameter and the 1D models leads to a further decomposition of the pressure drop contributions.

The modelling phase involves an iterative process which consists of the following steps:

(i) The pressure drop across the outlet duct of the DPF in the steady flow test rig is considered to be zero as initial assumption.

(ii) With this assumption, the lumped parameter model is applied to obtain a first raw value of the coefficient $a$ from the ratio between the pressure drop and the volumetric flow rate.

(iii) The porous wall permeability is calculated by means of equation (6), i.e. from the value of the coefficient $a$, the monolith geometry and the gas viscosity.

(iv) The 1D wall-flow DPF model is applied using the porous wall permeability as input data. The modelling includes the inlet duct, which has been previously characterised with OpenWAM, and the outlet duct of the experimental set-up to fit the measured pressure drop. The objective is to determine the value of the discharge coefficients defining the inertial contributions to the pressure drop in the canned DPF. It requires an iterative process with OpenWAM that converges when the volumetric flow rate and the pressure drop across the DPF and the outlet duct agree with measurements. The tuning of the discharge coefficients is performed on the basis of a number of criteria related to the expected qualitative distribution of the pressure drop across a clean wall-flow monolith, as found in the literature:

- The pressure drop due to local flow contraction at the channel inlet is about half the pressure drop due to local expansion at the channel outlet at high Reynolds number (Re). As Re diminishes, the ratio between the local contraction and expansion becomes lower [19, 25].

- The non-inertial pressure drop (friction and flow through the porous medium) contributes to around 50\% of the monolith pressure drop at high Re. As Re decreases, the non-inertial pressure drop becomes the dominant factor with a contribution of about $90 \%$ of the monolith pressure drop [19, 25].

The application of these criteria reduces the variety of possible combinations for the discharge coefficients values yielding the desired flow conditions in the system. Therefore it avoids from solutions without physical meaning. The criteria are completed assuming that the inertial pressure drop at the inlet cone is a loss of dynamic pressure, which is characterised by a discharge coefficient higher than 1 [26]. In addition, the vena contracta controls the pressure drop across the interface between the outlet cone and the outlet duct.

(v) Once the discharge coefficients are determined, the agreement between the calculated value of the pressure drop across the outlet duct and that assumed at the beginning of the iteration is checked. If there is not agreement, the last calculated value of the pressure drop at the outlet duct is subtracted to the measured pressure drop across the DPF and the outlet duct in order to estimate the pressure drop across the DPF. This new value is used to evaluate again the coefficient $a$ to be applied in the next iteration. The iterative process continues until the convergence of the pressure drop across the outlet duct is achieved. 
When the iterative procedure converges, the permeability of the clean porous wall is determined. Furthermore, the 1D wall-flow DPF model provides the value of the discharge coefficients, so that the characterisation of the inertial contributions to the pressure drop is also completed.

\subsection{Results and discussion}

By means of the iterative procedure described in section 2.4 a permeability equal to $2.485 \times 10^{-13} \mathrm{~m}^{2}$ was determined for the DPF whose experimental data are shown in Figure 2. The calculated discharge coefficients describing the inertial contributions to the pressure drop are also summarised in Table 2. A good agreement with experiments was obtained applying these data into the 1D DPF model. Figure 5 shows that the error is limited to $\pm 50 \mathrm{~Pa}$.

The modelled pressure drop across the outlet duct due to friction is also represented in Figure 5. Its value ranges between $5 \%$ and $7 \%$ of the measured pressure drop across the DPF and the outlet duct. The magnitude of this pressure drop demonstrates the importance of its calculation by means of the $1 \mathrm{D}$ model in order to obtain an accurate prediction of the permeability.

The analysis of the modelled results allows the assessment of the pressure drop contributions as a function of Re. Figure 6(a) shows the contributions to the pressure drop across the monolith, which are consistent with the described criteria. These results enforce the pressure drop decomposition shown in Figure 6(b), which is devoted to the pressure drop across the canned DPF. For the characterised DPF, the pressure drop in the inlet cone was of the same order of magnitude than the pressure drop in the monolith channels outlet. The same conclusion was obtained comparing the pressure drop in the outlet cone of the DPF with respect to the pressure drop in the monolith channels inlet. Such results indicate that the pressure drop decomposition from both experimental and modelled data may contribute to define guidelines for the optimum design of the DPF in function of the engine operating conditions and the exhaust line architecture, so that the fuel consumption penalty may be reduced and even the engine acoustic emissions mitigated [20].

Tests under impulsive flow were also performed to extend the validation of the proposed procedure to determine the porous wall permeability and the inertial pressure drop components. These tests allow to have a better comprehension of the impulsive response of the DPF. Experimental data were obtained testing the DPF in an impulse test rig. This facility allows the generation of engine-representative pressure pulses, which can be isolated in the time domain so that the reflected and transmitted pulses from the tested device can be analysed. The pressure pulses were measured with piezoelectric sensors. The characteristics of the set-up and the measurement procedure have been deeply described in [27].

Figure 7 shows the comparison between measured and modelled data under impulsive flow conditions at room temperature. The experiments were simulated by the model imposing the measured incident pressure pulse to the DPF, which is plotted with dashed-line.

Figures 7(a) and (b) show the time domain response of the DPF against two excitations of different peak amplitude (80 and $165 \mathrm{mbar}$ ) and duration (16 and $25 \mathrm{~ms}$ ). These plots give direct information about the characteristics of the 
attenuation that might be expected from the DPF in the exhaust system of engines. In addition, these results evidence the high sensitivity of the model to variations of the excitation.

The transmitted pulse by the DPF was well reproduced by the model, with only scarce differences in the interval of maximum pulse amplitude. Regarding the reflected pulse, the prediction of the discontinuity and the pressure reduction due to the sudden expansion of the flow at the inlet of the DPF was predicted with good accuracy. However, the pressure level was slightly underestimated in the overpressure region, which is controlled by the inlet channel plug.

The good agreement observed between experimental and modelled data under high unsteady flow conditions confirms the suitability of the proposed methodology to determine the permeability and the distribution of the inertial contributions to the pressure drop based on experimental characterisation at steady flow.

This conclusion is supported by the analysis of the results in the frequency domain, which are shown in Figure 8. Some differences are observed in both the transmitted and reflected SPL at high frequency, specially as the amplitude and duration of the pulses were reduced. Nevertheless, the proposed distribution of pressure drop across the DPF by means of the discharge coefficients and the definition of the porous wall permeability provides a very good prediction of the DPF response for frequencies below $400 \mathrm{~Hz}$. This prediction is acceptable up to $800 \mathrm{~Hz}$ at room temperature. Taking into account that the spectra are function of the frequency to speed of sound ratio $(f / a)$ [28], at $673 \mathrm{~K}$ these limits in frequency would increase up to $600 \mathrm{~Hz}$ and $1200 \mathrm{~Hz}$ respectively, due to the variation of the speed of sound with the temperature. This range ensures good predictions on engine operation since it includes the engine dynamic response in regular conditions.

\section{Slip-flow effect and pore structure}

\subsection{Relation between specific permeability and pore structure}

The main requirements for uncoated DPF filters, as that analysed in section 2, are high soot filtration efficiency and accumulation capacity, being the regeneration strategies based on fuel additives. Consequently, the absence of the catalyst in the porous medium leads to lower porosity and smaller mean pore diameter than in other DPF sorts. The porosity ranges between 0.4 and 0.45 whereas the mean pore diameter varies from $10 \mu \mathrm{m}$ to $20 \mu \mathrm{m}$ in uncoated DPFs [29].

Both the porosity and the mean pore diameter are related to the specific permeability. The specific permeability is a macroscopic parameter of the porous medium that is independent of both fluid properties and flow mechanisms. It is a function of the equivalent grain diameter and the porosity $(\varepsilon)$.

The equivalent grain diameter is defined as the mean diameter of the medium collector unit $\left(d_{c}\right)$, whereas the relation with the porosity is expressed through a porosity factor $(f(\varepsilon))$. The general expression for the evaluation of the specific permeability is: 


$$
k_{w}=f(\varepsilon) d_{c}^{2}
$$

On the other hand, the mean pore diameter is frequently used to describe the porous wall structure. It is defined as a hydraulic diameter, so that it represents the diameter of a sphere conserving the wet surface area of the substrate. The mean pore diameter in a packed bed of spherical particles, which is representative of the wall-flow DPF monolith substrate, can be obtained as a function of the porosity and the collector unit diameter:

$$
d_{p}=\frac{4 V_{\text {void }}}{A}=\frac{4 \frac{\pi}{6} d_{c}^{3} \frac{\varepsilon}{1-\varepsilon}}{\pi d_{c}^{2}}=\frac{2}{3} \frac{\varepsilon}{1-\varepsilon} d_{c}
$$

The porosity factor is not an universal function and has been developed by several authors from different approaches. Some of the most remarkable models for packed beds of spherical particles are listed in Table 3. Figure 9 shows the value of the porosity factor predicted by every model cited in Table 3 in a range of porosity frequently used in honeycomb wall-flow monolith filters.

Nevertheless, the value of the permeability determined with the procedure described in section 2 is not the specific permeability. In order to determine the specific permeability two considerations must be previously remarked:

(i) Slip-flow effect. The flow velocity at the solid walls of the conduits of the porous medium is different to zero. Therefore, equation (11) must be corrected to incorporate this effect by means of the Stokes-Cunningham factor (SCF), as:

$$
k_{w}=f(\varepsilon) d_{c}^{2} S C F
$$

The Stokes-Cunningham factor is a function of the Knudsen number $(\mathrm{Kn})$ relative to the mean pore diameter:

$$
\begin{gathered}
S C F=1+K n\left(1.257+0.4 e^{\frac{-1.1}{K n}}\right) \\
K n=\frac{2 \phi}{d_{p}}
\end{gathered}
$$

Figure 10 shows graphically the effect of the mean pore diameter and the flow temperature on the SCF. Its value increases with the flow temperature, since the variation of the mean free path of the gas molecules $(\phi)$ affects the Knudsen number. As described in section 2.2, tests in the steady flow test rig were conducted at room temperature $(297.5 \mathrm{~K})$. The SCF value varies at this temperature between 1.0083 and 1.0167 when the mean pore diameter decreases from $20 \mu \mathrm{m}$ to $10 \mu \mathrm{m}$.

(ii) The direct application of the porosity factor models for packed beds of spherical particles does not yield the permeability obtained in section 2, even considering the slip-flow correction, as Figure 11 shows. The reasons for these results obey to three different causes: 
a) The flow inside the porous medium has been supposed to travel a distance equal to the wall thickness. This assumption is applied to both models described in section 2. However, the effective length of the flow path is longer than the wall thickness because of the pore connections. It affects to the interstitial velocity and to the number of pores in parallel to match the porosity [36]. This concept is assessed by the tortuosity factor, which is not a property of the porous medium but a parameter of 1D models of the porous medium [36]. The permeability obtained empirically contains this discrepancy and becomes underestimated because the lumped parameter and the 1D DPF models applied in this study do not include a correction to consider the tortuosity.

b) The existence of parallel-type pore non-uniformities. The fluid tends to flow through the larger pores, so that the permeability is essentially controlled by them [36]. The existence of this phenomenon causes a deviation of the definition of both the size distribution and the spherical shape of the pores. This fact induces models to predict an underestimated permeability due to the consideration of a mean pore diameter.

c) The effect of serial-type pore non-uniformities. It is caused by the variation of the effective cross-section of every conduit along the length of the flow path. Since the permeability is controlled by the smallest effective cross-section of the conduit flow, the consideration of a mean pore diameter induces models to overpredict the permeability.

Parallel and serial-type pore non-uniformities are phenomena affecting in opposite way and therefore mutual cancellation could be expected. Nevertheless, the three effects might be considered to obtain the specific permeability of the porous medium.

According to this discussion, Konstandopoulos [14] reported that a tortuosity factor equal to 3.25 must be considered. This correction coefficient was obtained with a lumped parameter DPF model similar to that proposed in section 2.1. Its use led to fit well the empirical data of a large sample of cordierite and $\mathrm{SiC}$ made wall-flow monoliths to the Carman-Kozeny's and the Kuwabara's models.

Applying this tortuosity correction, the continuum permeability of the characterised DPF becomes $8.076 \cdot 10^{-13} \mathrm{~m}^{2}$. This value includes the slip-flow effect and therefore, it does not define the specific permeability.

The slip-flow effect cannot be evaluated unless the mean pore diameter is known. Therefore, the specific permeability cannot be determined because the mean pore diameter has been not still obtained.

In order to define the mean pore diameter and the porosity of the porous substrate, Figure 12 shows the value of the continuum permeability obtained applying the Kuwabara's porosity factor model. The temperature was considered the same as in the steady flow tests to calculate the slip-flow effect.

There are several combinations in the values of porosity and mean pore diameter able to yield the obtained value of the continuum permeability, which is plotted with black dashed-line in Figure 12. Confident solutions can be attained if the porosity or the mean pore diameter are known. However, it is possible that neither the porosity nor the mean pore diameter are trustworthy. In this case an additional condition is required: 
- Procedure 1: Figure 12(a) shows a plot of the continuum permeability against the porosity for the mean pore diameter ranging between $10 \mu \mathrm{m}$ and $15 \mu \mathrm{m}$. In spite of the fact that this range is lower than that found in the literature for this sort of DPF $([10,20] \mu \mathrm{m})$, there is not solution to define the porosity range. The upper limit is not determined because the Kuwabara's porosity factor and a mean pore size diameter of $10 \mu \mathrm{m}$ do not cross the desired permeability for any porosity. The lower porosity limit, which is given by $d_{p}=15 \mu \mathrm{m}$, is 0.25 . It is clearly far from the data available in the literature for uncoated filters. Therefore, this procedure does not allow to determine a porosity value coherent with already existing knowledge of the order of magnitude that define the porous substrate. Similar results would be obtained in the case of applying the Happel's model. The Rumpf \& Gupte, Blake-Kozeny and Karman-Kozeny's models would provide a rough porosity range, but still out of the literature data range. Therefore, the solution to which Procedure 1 leads would be of high uncertainty, if any.

- Procedure 2: Figure 12(b) shows a plot of the continuum permeability as a function of the mean pore diameter. The porosity ranges between 0.35 and 0.5 . This range corresponds to a small variation of the mean pore diameter, between $11.3 \mu \mathrm{m}$ and $12.8 \mu \mathrm{m}$, that match the desired continuum permeability. The mean value from the range shown in Figure 12(b) is chosen as mean pore diameter value, which is equal to $12 \mu \mathrm{m}$ applying the Kuwabara's porosity factor. With this mean pore diameter and applying equation (13), the porous wall porosity results equal to 0.415 to agree the continuum permeability.

It is important to remark that the obtained range for the mean pore diameter is very reduced in spite of the wide calculation range for porosity, which has been even further than the literature order of magnitude in this sort of DPF $([0.35,0.5]$ vs. $[0.4,0.45])$. It highlights the high certainty in the solution of the mean pore diameter.

In conclusion, the higher consistency shown by Procedure 2 indicates that, if the permeability is known, the determination of the pore structure must be based on the definition of a suitable porosity range. These data give a tight range for the determination of the mean pore diameter, from which a estimation of the porosity can finally be obtained. Figure 13 shows the plots of the results obtained by this procedure considering different models to calculate the porosity factor in packed beds of spherical particles. These results are also summarised in Table 4 and in Figure 14, which represents the obtained solution $\left(d_{p}, \varepsilon\right)$ on the iso-line $k_{w}=8.076 \cdot 10^{-13} \mathrm{~m}^{2}$.

The differences between the porosity factor models are almost negligible and the dispersion in the solution is also low; the mean pore diameter is inside the range $[11.3,12.4] \mu \mathrm{m}$ and the porosity falls into [0.405, 0.415]. These results contributes to guarantee the consistency of the proposed methodology.

The solution from the Kuwabara's model was considered the most representative of the pore structure, and hence it was applied in the fluid dynamic analysis of the DPF response. The reason lies on the widespread use of the Kuwabara's model in the simulation of the filtration process in packed beds of spherical particles through the unit collector filtration theory [37-39]. 
Therefore, known the value of the mean pore diameter $(12 \mu \mathrm{m})$, the evaluation of the Stokes-Cunningham factor at test temperature is also possible. According to equation (14), the SCF value is 1.0139 for tests discussed in section 2. Finally, dividing the continuum permeability by SCF, the specific permeability of the porous wall results equal to $7.965 \cdot 10^{-13} \mathrm{~m}^{2}$. The value of the main properties defining the pore structure are summarised in Table 5.

\subsection{Influence of the slip-flow effect}

The influence of the slip-flow effect in coated DPFs has been widely proved to be relevant [38, 40]. Experiments with the DPF were performed in an engine test bench in order to assess the significance of this phenomena in uncoated filters with typical wall micro-structural properties. In these tests, the high gas temperature varies the permeability due to the slip-flow effect, which is also function of the mean pore diameter, as shown in Figure 10 and equations (14) and (15).

The measured instantaneous pressure upstream and downstream of the DPF and the isentropic instantaneous temperature upstream of it were imposed to the model. The isentropic instantaneous temperature was applied to approximate the instantaneous temperature. It was obtained from the instantaneous pressure and the measured mean pressure and temperature. Since the pressure drop is imposed, the control parameters for the modelling are the air mass flow and the temperature downstream of the DPF. They both were compared with the measured mean values.

Every operating point, identified by the engine speed, was tested with a very low equivalence ratio, ranged between 0.074 at $900 \mathrm{rpm}$ and 0.169 at $2000 \mathrm{rpm}$ to avoid any influence of the DPF soot loading. As drawback, this strategy also limited the test temperature below regular engine operation.

As Figure 15(a) shows, a good agreement between the measured data and the calculated results was obtained for all tested operating points when the slip-flow effect was considered. These results highlights the capabilities of the proposed methodology to describe both the pressure drop contributions and the pore structure in wall-flow DPFs. The prediction of the gas temperature at the outlet of the DPF was performed with a nodal heat transfer model. The model solves the heat transfer equation both in the axial and radial direction of the monolith channels and also includes the radial heat transfer from the monolith to the metallic shell and to the environment [18].

The plot in Figure 15(b) represents the instantaneous value of the continuum permeability calculated at $2000 \mathrm{rpm}$. The differences found in the value of the continuum permeability depending on the radial location evidence the effect of the gas temperature in the mean free path of the gas molecules. On the other hand, the differences with respect to the specific permeability, represented by the dashed-line, reach to values higher than $3 \%$ in the core region of the monolith, whereas in the peripheral region the minimum difference is $2.5 \%$. This difference would increase during operation at higher temperature. Nevertheless, its magnitude is high enough to show that if the slip-flow effect is not considered, the mass flow prediction across the DPF would be underestimated, as Figure 15(a) confirms. Nevertheless, the error is very low at low temperature, as concluded from previous modelling under steady and impulsive flow at room temperature. As the gas temperature increases, the error in mass flow of the model also increases monotonically, 
so that the maximum error in mass flow is obtained at the highest temperature of the test range $(487 \mathrm{~K})$ and reaches $1.6 \%$ of the measured value.

It is important to note that previous results have confirmed that the model does not show numerical error. So, when the model takes into account the slip flow effect, which is an important effect at high temperature, it is reached a more detailed description of the physics and the error versus experimental data is reduced without any artificial correction. It also certifies a previous good evaluation of the mean pore diameter, from which depends an accurate evaluation of the SFC.

\section{Summary and conclusions}

An experimental-theoretical methodology has been presented for the assessment of the pore structure properties and the pressure drop contributions in honeycomb wall-flow monolith filters. The methodology is based on the measurement of the pressure drop across the DPF in a cold steady flow test rig. In order to decompose every contribution to the pressure drop, tests were modelled with a lumped parameter and a 1D wall-flow DPF models. As a result, the continuum permeability of the porous wall has been firstly determined.

Known the continuum permeability, the porosity and the mean pore diameter have been quantified. To do this, several porosity factor models for packed beds of spherical particles have been considered. According to the obtained results, a first definition of the porosity range is advised. From this, a narrow range for the mean pore diameter is obtained and leads to define the value of the mean pore diameter with high accuracy. Subsequently, the value of the porosity can be estimated.

Similar results in the calculation of both the mean pore diameter and the porosity were obtained with all the porosity factor models considered. The model proposed by Kuwabara was adopted in this work as solution in order to maintain the consistency with the formulation of most widely-used filtration models for packed beds of spherical particles. Finally, the value of the mean pore diameter permits the assessment of the slip-flow correction and leads to determine the specific permeability of the porous wall substrate.

A set of experiments and their corresponding modellings were performed to confirm the accuracy and reliability of the determined pore structure properties. These tests are related to the fluid dynamic characterisation of the DPF in order to analyse its influence on the engine performance. Impulsive flow tests have shown the suitability of both the permeability and the distribution of the inertial pressure drop determination even under unsteady flow conditions. Hot pulsating flow tests, which were carried out in an engine test bench, have included the effect of the flow temperature on the permeability through the slip-flow effect, which also strongly depends on the definition of the mean pore diameter. These tests have been modelled imposing the experimental pressure drop across the tested system. The comparison between the experimental data and the modelling results has shown the importance of the slip-flow effect on the air mass flow prediction across uncoated wall-flow monoliths. 


\section{Appendix A: Formulation of the 1D wall-flow DPF model}

The 1D wall-flow DPF model solves the flow across the canned DPF device. The governing equations are formulated for non-homentropic one-dimensional unsteady compressible flow inside the monolith channels:

- Mass conservation

$$
\frac{\partial\left(\rho_{i} F_{i}\right)}{\partial t}+\frac{\partial\left(\rho_{i} u_{i} F_{i}\right)}{\partial x}=(-1)^{i} 4\left(\alpha-2 w_{p} i\right) \rho_{i} u_{w_{i}}
$$

- Momentum conservation

$$
\frac{\partial\left(\rho_{i} u_{i} F_{i}\right)}{\partial t}+\frac{\partial\left(\rho_{i} u_{i}^{2} F_{i}+p_{i} F_{i}\right)}{\partial x}-p_{i} \frac{d F_{i}}{d x}=-F_{w} \mu_{i} u_{i}
$$

- Energy conservation

$$
\frac{\partial\left(e_{0 i} \rho_{i} F_{i}\right)}{\partial t}+\frac{\partial\left(h_{0 i} \rho_{i} u_{i} F_{i}\right)}{\partial x}=q_{i} \rho_{i} F_{i}+(-1)^{i} 4\left(\alpha-2 w_{p} i\right) h_{0 w} \rho_{i} u_{w_{i}}
$$

Subscript $i$ in equations (16), (17) and (18) identifies the type of monolith channel. It takes value 0 for the solution of the outlet channels and value 1 in the case of the inlet channels.

The conservation equations system in a pair of inlet and outlet channels is closed with the addition of the state equation for an ideal gas in every of the channels and the equation governing the pressure drop across the porous medium. It allows to obtain the value of the filtration velocity.

At this work, the Forchheimer's effect has been neglected because of the laminar flow inside the channels. Therefore, the pressure drop is governed by the Darcy's equation. The filtration velocity at every node in the axial direction of the channel is obtained in the most general case as

$$
\begin{gathered}
u_{w_{1}}=\frac{\Delta p}{\frac{\mu w_{w}}{k_{w}} \frac{\rho_{1}\left(\alpha-2 w_{p}\right)}{\rho_{0} \alpha}+\frac{\mu\left(\alpha-2 w_{p}\right)}{2 k_{p}} \ln \left(\frac{\alpha}{\alpha-2 w_{p}}\right)} \\
u_{w_{0}}=\frac{u_{w_{1}} \rho_{1}\left(\alpha-2 w_{p}\right)}{\rho_{0} \alpha}
\end{gathered}
$$

where $\Delta p$ is the pressure drop between the inlet and the outlet channel, which is obtained from the solution of the conservation equations, as suggested also in Figure 16. This solution has been obtained assuming that the filtration velocity may vary between the porous wall surface of the inlet channels and that of the outlet channels. The reason is the possible existence of the particulate layer and the assumed linear variation of the channel width represented in Figure 16.

\section{Acknowledgements}

This work has been partially supported by the spanish Ministerio de Ciencia e Innovación through grant number DPI2010-20891-C02-02. 
[1] Solvat O, Marez P, Belot G. Passenger car serial application of a particulate filter system on a common-rail, direct-injection Diesel engine. In: SAE Technical Paper 2000-01-0473; 2000.

[2] Knecht W. Diesel engine development in view of reduced emission standards. Energy 2008;33(2):264-271.

[3] Torregrosa AJ, Broatch A, Novella R, Mónico LF. Suitability analysis of advanced diesel combustion concepts for emissions and noise control. Energy 2011;36(2):825-838.

[4] Rakopoulos CD, Dimaratos AM, Giakoumis EG, Rakopoulos DC. Evaluation of the effect of engine, load and turbocharger parameters on transient emissions of Diesel engine. Energy Conversion and Management 2009;50(9):2381-2393.

[5] Rakopoulos CD, Dimaratos AM, Giakoumis EG, Rakopoulos DC. Investigating the emissions during acceleration of a turbocharged Diesel engine operating with bio-diesel or n-butanol diesel fuel blends, Energy 2010;35(12):5173-5184.

[6] Sarvi A, Zevenhoven R. Large-scale Diesel engine emission control parameters. Energy 2010;35(2):1139-1145.

[7] Bermúdez V, Luján JM, Pla B, Linares WG. Effects of low pressure exhaust gas recirculation on regulated and unregulated gaseous emissions during NEDC in a light-duty Diesel engine. Energy 2011;36(9):5655-5665.

[8] Regulation (EC) No 715/2007 of the European Parliament and of the Council of 20 June 2007 on type approval of motor vehicles with respect to emissions from light passenger and commercial vehicles (Euro 5 and Euro 6) and on access to vehicle repair and maintenance information, Official Journal of the European Union (June 2007).

[9] Zervas E, Poulopoulos S, Philippopoulos C. CO2 emissions change from the introduction of diesel passenger cars: Case of Greece. Energy 2006;31(14):2915-2925.

[10] Kittelson DB. Engines and nanoparticles, a review. Journal of Aerosol Science 1998;29(5-6):575-588.

[11] Konstandopoulos AG. Update on simulation of diesel particulate emission control systems. Ulysses - Modelling and Simulation Workshop. The future propulsion as One System. Cracow, Poland; May 2009.

[12] Bisset EJ. Mathematical model of the thermal regeneration of a wall-flow monolith diesel particulate filter. Chemical Engineering Science 1984;39(7-8):1233-1244.

[13] Murtagh M. Diesel particulate filters (DPF): A short course. In: Diesel particulate and NQ emissions course. Ann Arbor, MI: University of Leeds; 2002.

[14] Konstandopoulos AG. Flow resistance descriptors for diesel particulate filters: Definitions, measurements and testing. In: SAE Technical Paper 2003-01-0846; 2003.

[15] Haralampous OA, Kandylas IP, Koltsakis GC, Samaras ZC. Diesel particulate filter pressure drop. Part 1: Modelling and experimental validation. International Journal of Engine Research 2004;5:149-162.

[16] Yapaulo RA, Wirojsakunchai E, Orita T, Foster DE, Akard M, Walker LR et al. Impact of filtration velocities and particulate matter characteristics on diesel particulate filter wall loading. International Journal of Engine Research 2009;10(5):287-304.

[17] Piscaglia F, Ferrari G. A novel 1D approach for the simulation of unsteady reacting flows in diesel exhaust after-treatment systems. Energy 2009;34:2051-2062.

[18] Bermúdez V, Serrano JR, Piqueras P, García-Afonso O. Assessment by means of gas dynamic modelling of a pre-turbo diesel particulate filter configuration in a turbocharged HSDI Diesel engine under full-load transient operation. Proceedings of the Institution of Mechanical Engineers, Part D: Journal of Automobile Engineering 2011;225(9):1134-1155.

[19] Konstandopoulos AG, Skaperdas E, Masoudi M. Inertial contributions to the pressure drop of diesel particulate filters. In: SAE Technical Paper 2001-01-0909; 2001.

[20] Torregrosa AJ, Serrano JR, Arnau FJ, Piqueras P. A fluid dynamic model for unsteady compressible flow in wall-flow diesel particulate filters. Energy 2011;36(1):671-684.

[21] Desantes JM, Serrano JR, Arnau FJ, Piqueras P. Derivation of the method of characteristics for the fluid dynamic solution of flow advection along porous wall channels. Applied Mathematical Modelling 2011, doi:10.1016/j.apm.2011.09.090.

[22] Openwam website. CMT-Motores Térmicos (Universitat Politècnica de València). www.openwam.org (2011). 
[23] Payri F, Galindo J, Serrano JR, Arnau FJ. Analysis of numerical methods to solve one-dimensional fluid-dynamic governing equations under impulsive flow in tapered ducts. International Journal of Mechanical Science 2004;46(7):981-1004.

[24] Benson RS. The thermodynamics and gas dynamics of internal-combustion engines, Vol. 1. Clarendon Press, New York; 1982.

[25] Masoudi M. Hydrodynamics of diesel particulate filters. In: SAE Technical Paper 2002-01-1016; 2002.

[26] Payri F, Reyes E, Galindo J. Analysis and modeling of the fluid-dynamic effects in branched exhaust junctions of ICE. Journal of Engineering for Gas Turbine and Power - Transactions of the ASME 2001;123(1):197-203.

[27] Payri F, Desantes JM, Broatch A. Modified impulse method for the measurement of the frequency response of acoustic filters to weakly nonlinear transient excitations. Journal of the Acoustical Society of America 2000;107(2):731-738.

[28] Desantes JM, Torregrosa AJ, Broatch A, Moya D. Development of time-domain models for automotive exhaust muffers. Acoustique et Techniques 2003;31:21-26.

[29] Wall-flow monoliths. DieselNet Technology Guide. www.dieselnet.com (September 2005).

[30] Rumpf H, Gupte AR. Einfüsse und Korngrössenverteilung in Widerstandsdesetz der Porenströmung. Chemie Ingenieur Technik 1971;43:367375.

[31] Blake FC. The resistance of packing to fluid flow. Trans. American Institute of Chemical Engineering 1922;14:415-421.

[32] Kozeny J. Über kapillare Leitung des Wassers in Boden. Sitzungsberichte der Akademie der Wissenschaften in Wien 1927;136:271-306.

[33] Carman PC. Fluid flow through a granular bed. Chemical Engineering Research and Design 1937;15:150-156.

[34] Happel J. Viscous flow in multiparticle systems: Slow motion of fluids relative to beds of spherical particles. American Institute of Chemical Engineers Journal 1958;4:197-201.

[35] Kuwabara S. The forces experienced by randomly distributed parallel circular cylinders or spheres in a viscous fluid at small Reynolds numbers. Journal of the Physical Society of Japan 1959;14:527-532.

[36] Dullien FAL. Porous Media. Fluid Transport and Pore Structure. Academic Press, New York; 1992.

[37] Lee KW, Gieseke JA. Collection of aerosol particles by packed beds. Environmental Science and Technology 1979;13(4):466-470.

[38] Konstandopoulos AG, Kostuglou M, Skaperdas E, Papaioannou E, Zarvalis D, Kladopoulou E. Fundamental studies of diesel particulate filters: Transient loading, regeneration and aging. In: SAE Technical Paper 2000-01-1016;2000.

[39] Mohammed H, Triana AP, Yang SL, Johnson JH. An advanced 1D 2-layer catalized diesel particulate filter model to simulate: filtration by the wall and particulate cake, oxidation in the wall and particulate cake by $\mathrm{NQ}_{2}$ and $\mathrm{O}_{2}$ and regeneration by heat addition. In: SAE Technical Paper 2006-01-0467;2006.

[40] Kwetkus BA, Egli W. The monolithic ceramic filter module: Filtration properties and DeNQ potential. International Ceramic Review 1999;48(5):314-321.

\title{
Nomenclature
}

\author{
A wet surface in a packed bed of spherical particles $\left(\mathrm{m}^{2}\right)$ \\ $d_{c} \quad$ mean collector unit diameter $(\mathrm{m})$ \\ $d_{p} \quad$ mean pore diameter $(\mathrm{m})$ \\ $e_{0} \quad$ specific stagnation internal energy $(\mathrm{J} / \mathrm{kg})$ \\ $f(\varepsilon) \quad$ porosity factor function \\ $F \quad$ cross-section area $\left(\mathrm{m}^{2}\right)$ \\ $F_{w} \quad$ momentum transfer coefficient for square channels \\ $h_{0} \quad$ specific stagnation enthalpy $(\mathrm{J} / \mathrm{kg})$
}




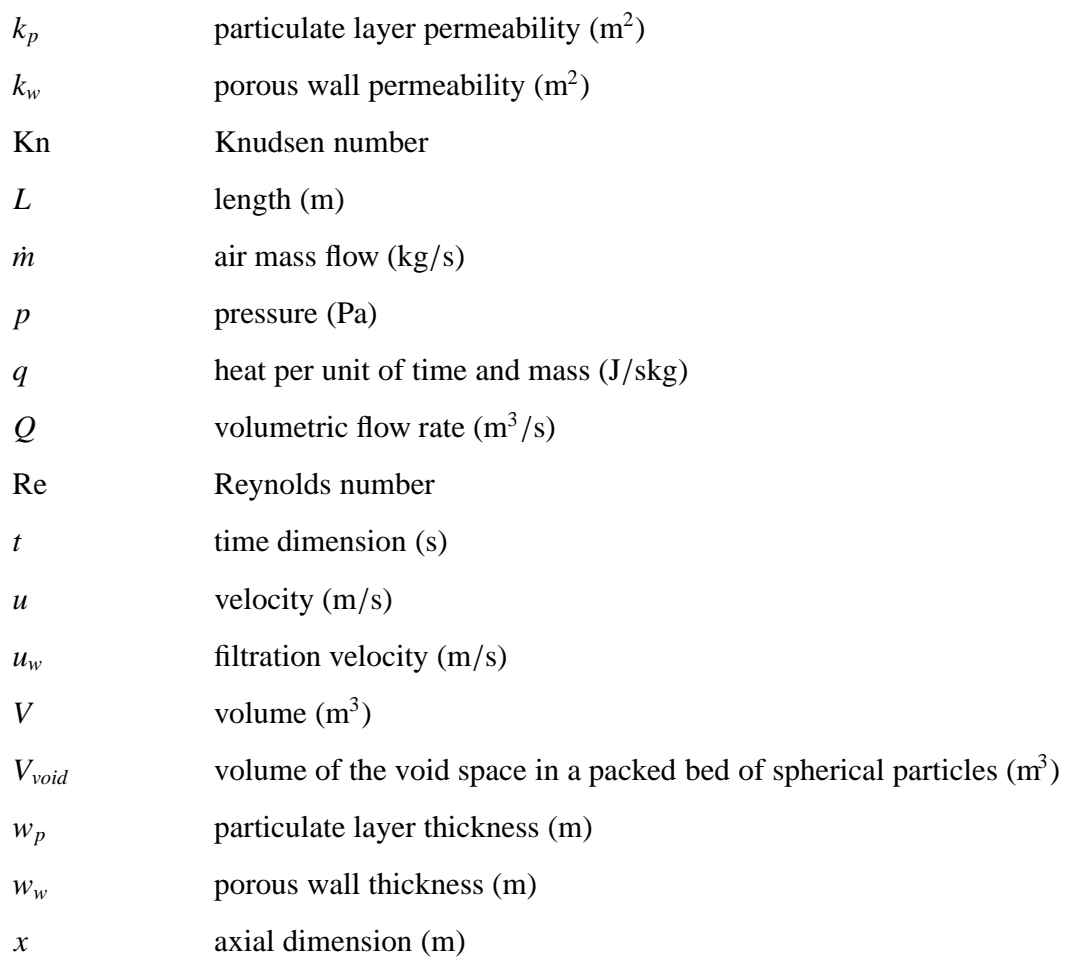

\section{Greek letters}

$\alpha$

$\beta_{F}$

$\Delta p$

$\varepsilon$

$\mu$

$\phi$

$\rho$

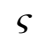

\section{Subscripts}

$i$

ie

id

mon

oc honeycomb cell size (m)

Forchheimer's coefficient $\left(\mathrm{m}^{-1}\right)$

stagnation pressure drop $(\mathrm{Pa})$

porosity

dynamic viscosity (sPa)

molecules mean free path (m)

density $\left(\mathrm{kg} / \mathrm{m}^{3}\right)$

pressure drop coefficient

$$
\text { index for type of channel }
$$

expansion at inlet cone

inlet duct

monolith

contraction at outlet cone 


\section{Abbreviations}

OD

zero-dimensional

1D

one-dimensional

DPF

diesel particulate filter

SCF

Stokes-Cunningham factor

SPL sound pressure level (dB) 


\section{List of Tables}

- Table 1. Geometrical characteristics of the DPF.

- Table 2. Value of the discharge coefficients for the characterisation of the inertial pressure drop across the DPF system.

- Table 3. Models for porosity factor in packed beds of spherical particles.

- Table 4. Determination of porosity and mean pore diameter for several porosity factor models.

- Table 5. Summary of the determined pore structure properties and the slip-flow correction at test temperature.

\section{List of Figures}

- Figure 1. Experimental set-up of the DPF in the steady flow test rig.

- Figure 2. Experimental pressure drop decomposition.

- Figure 3. DPF model scheme into the gas dynamics code OpenWAM ${ }^{\mathrm{TM}}$ : combination of $1 \mathrm{D}$ elements for monolith channels, OD elements for cones and boundary conditions..

- Figure 4. Procedure for determination of porous wall permeability and inertial pressure drop contributions in honeycomb wall-flow DPFs.

- Figure 5. Comparison between measured and modelled pressure drop across the DPF and the outlet duct when the iterative characterisation procedure is converged.

- Figure 6. Decomposition of the pressure drop contributions: a) Monolith; b) canned DPF.

- Figure 7. Experimental and modelled results in time domain under impulsive flow conditions.

- Figure 8. Experimental and modelled results in frequency domain under impulsive flow conditions.

- Figure 9. Porosity factor value for different flow models in packed beds of spherical particles.

- Figure 10. Stokes-Cunningham factor for slip-flow correction of the continuum permeability.

- Figure 11. Disagreement between empirical and calculated permeability as a function of several porosity factor correlations $(\varepsilon=0.4)$.

- Figure 12. Procedures for porosity and mean pore diameter determination from continuum permeability and Kuwabara's porosity factor.

- Figure 13. Permeability as a function of $d_{p}$ and $\varepsilon$ for several porosity factor models.

- Figure 14. Iso-lines of $k_{w}=8.076 \cdot 10^{-13} \mathrm{~m}^{2}$ for every porosity factor model and solution provided applying Procedure 2 .

- Figure 15. DPF response under hot pulsating flow conditions. a) Comparison between measured and predicted mass flow and downstream DPF temperature by the 1D wall-flow DPF model. b) Continuum permeability at two monolith radial locations at $2000 \mathrm{rpm}$ and comparison against specific permeability of the porous wall.

- Figure 16. Axial and cross-section geometry of channels in wall-flow DPF monoliths and description of the spatial mesh and filtration velocity through the porous medium. 
Table 1: Geometrical characteristics of the DPF.

\begin{tabular}{lcc}
\hline \hline Length & $\mathrm{m}$ & 0.2 \\
Diameter & $\mathrm{m}$ & 0.132 \\
Volume & $\mathrm{cm}^{3}$ & 2740 \\
Cross-section & $\mathrm{m}^{2}$ & 0.0137 \\
Plug length & $\mathrm{mm}$ & 3.2 \\
Cell density & $\mathrm{cpsi}$ & 200 \\
Filter cell size & $\mathrm{mm}$ & 1.486 \\
Wall thickness & $\mathrm{mm}$ & 0.31 \\
Number of channels & - & 4246 \\
Filtration area & $\mathrm{m}{ }^{2}$ & 2.5 \\
Inlet cone volume & $\mathrm{cm}^{3}$ & 500 \\
Outlet cone volume & $\mathrm{cm}^{3}$ & 450 \\
Inlet duct diameter & $\mathrm{m}$ & 0.05 \\
Outlet duct diameter & $\mathrm{m}$ & 0.047 \\
\hline \hline
\end{tabular}

Table 2: Value of the discharge coefficients for the characterisation of the inertial pressure drop across the DPF system.

\begin{tabular}{lcc}
\hline \hline Discharge coefficient & Boundary condition & Value \\
\hline DPF inlet cone & 1 & 1.55 \\
Monolith inlet & 2 & 0.6 \\
Monolith outlet & 5 & 0.53 \\
DPF outlet cone & 6 & 0.97 \\
\hline \hline
\end{tabular}


Table 3: Models for porosity factor in packed beds of spherical particles.

\begin{tabular}{lc}
\hline \hline Source & $f(\varepsilon)$ \\
\hline Rumpf \& Gupte [30] & $\frac{\varepsilon^{5.5}}{5.6}$ \\
Blake-Kozeny [31, 32] & $\frac{\varepsilon^{3}}{150(1-\varepsilon)^{2}}$ \\
Carman-Kozeny [33] & $\frac{\varepsilon^{3}}{180(1-\varepsilon)^{2}}$ \\
Happel [34] & $\frac{1}{18}\left(\frac{3-4.5(1-\varepsilon)^{\frac{1}{3}}+4.5(1-\varepsilon)^{\frac{5}{3}}-3(1-\varepsilon)^{2}}{(1-\varepsilon)\left(3+2(1-\varepsilon)^{\frac{5}{3}}\right)}\right)$ \\
Kuwabara [35] & $\frac{0.065\left(2-\frac{9}{5}(1-\varepsilon)^{\frac{1}{3}}-\varepsilon-\frac{1}{5}(1-\varepsilon)^{2}\right)}{1-\varepsilon}$ \\
\hline \hline
\end{tabular}

Table 4: Determination of porosity and mean pore diameter for several porosity factor models.

\begin{tabular}{lccc}
\hline \hline \multirow{2}{*}{ Model } & \multirow{2}{*}{$d_{p}$ range } & \multicolumn{2}{c}{ Solution } \\
& {$[\mu \mathrm{m}]$} & $d_{p}[\mu \mathrm{m}]$ & $\varepsilon[-]$ \\
\hline Kuwabara & {$[11.3,12.8]$} & 12 & 0.415 \\
Rumpf\&Gupte & {$[9.5,13.6]$} & 11.5 & 0.405 \\
Blake-Kozeny & {$[10.3,12.3]$} & 11.3 & 0.416 \\
Carman-Kozeny & {$[11.3,13.5]$} & 12.4 & 0.415 \\
Happel & {$[11,12.8]$} & 11.9 & 0.410 \\
\hline \hline
\end{tabular}

Table 5: Summary of the determined pore structure properties and the slip-flow correction at test temperature.

\begin{tabular}{lcc}
\hline \hline specific $k_{w}$ & {$\left[\mathrm{~m}^{2}\right]$} & $7.965 \cdot 10^{-13}$ \\
$\varepsilon$ & {$[-]$} & 0.415 \\
$d_{p}$ & {$[\mu \mathrm{m}]$} & 12 \\
$d_{c}$ & {$[\mu \mathrm{m}]$} & 25.4 \\
$\mathrm{SCF}$ & {$[-]$} & 1.0139 \\
\hline \hline
\end{tabular}




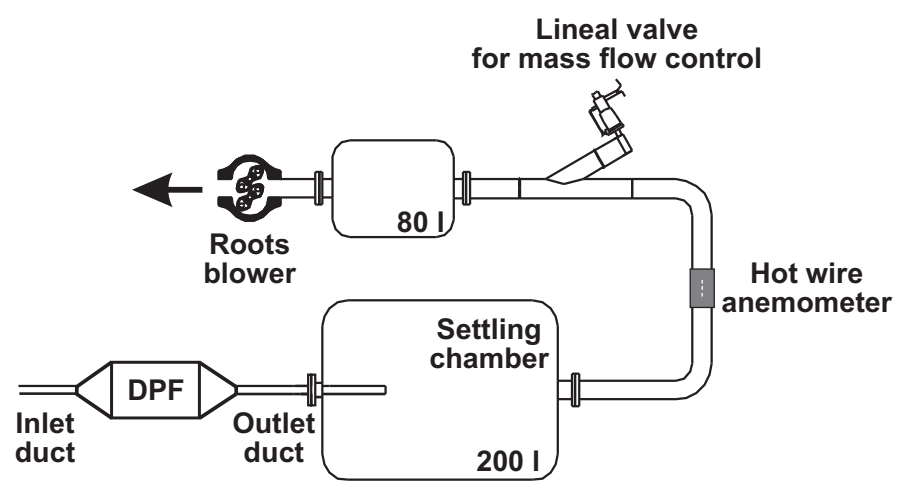

Figure 1: Experimental set-up of the DPF in the steady flow test rig.

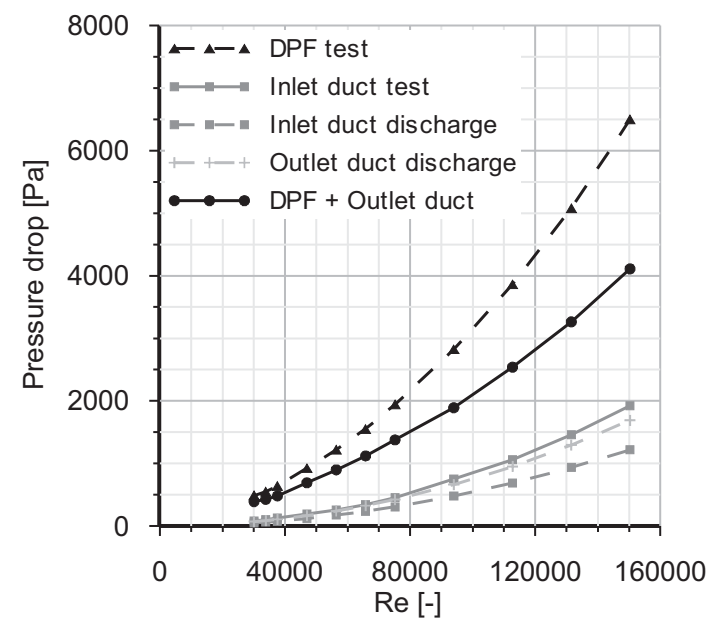

Figure 2: Experimental pressure drop decomposition.

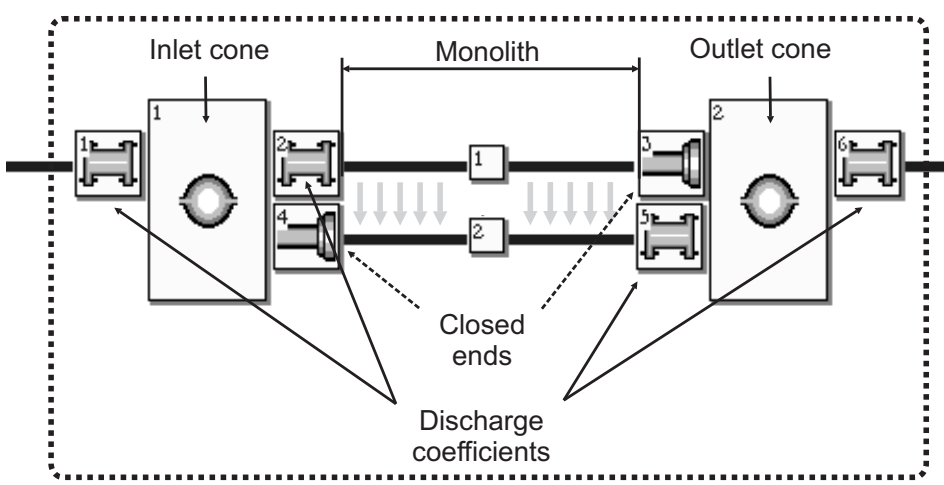

Figure 3: DPF model scheme into the gas dynamics code OpenWAM ${ }^{\mathrm{TM}}$ : combination of $1 \mathrm{D}$ elements for monolith channels, $0 \mathrm{D}$ elements for cones and boundary conditions. 


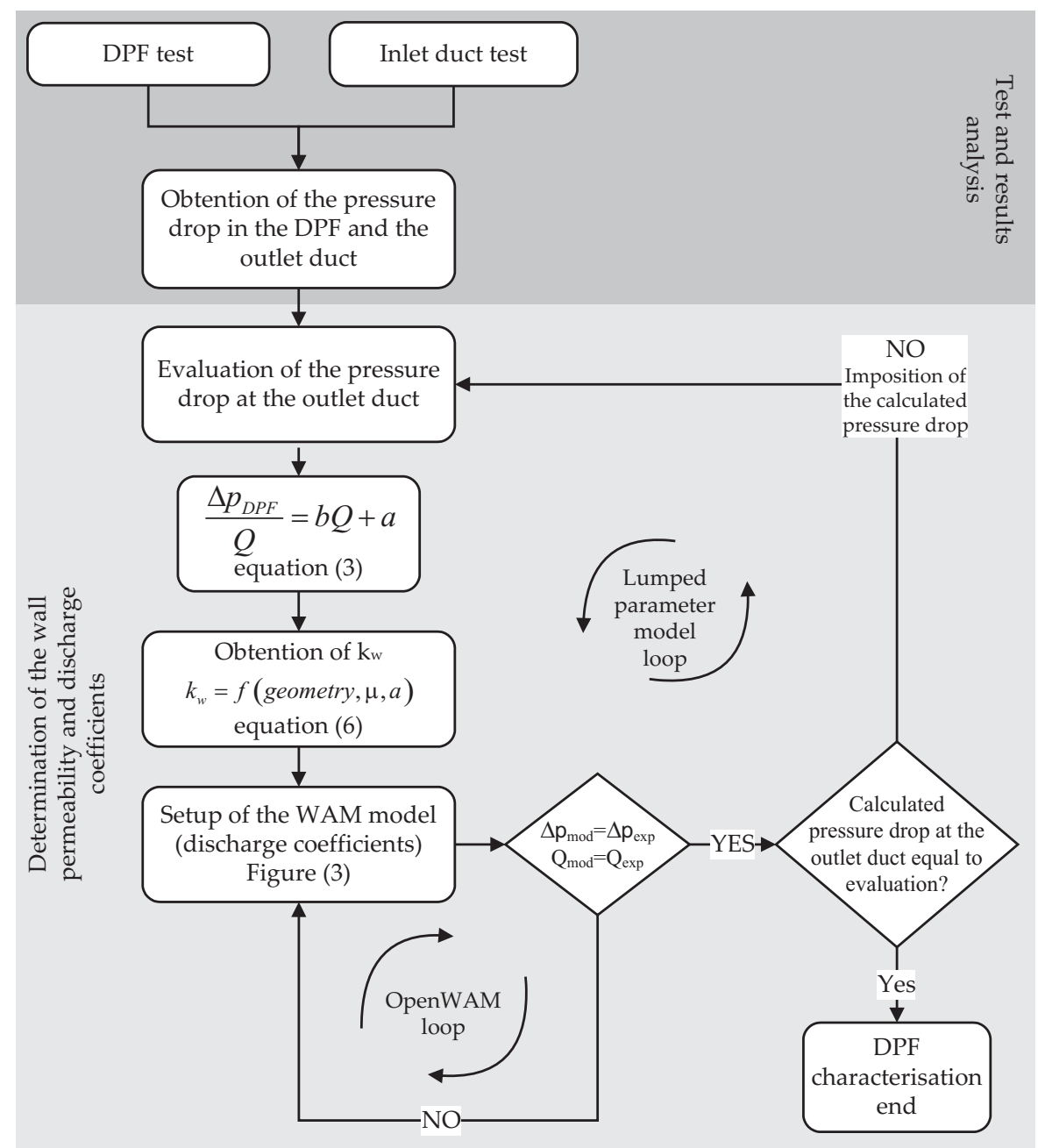

Figure 4: Procedure for determination of porous wall permeability and inertial pressure drop contributions in honeycomb wall-flow DPFs. 


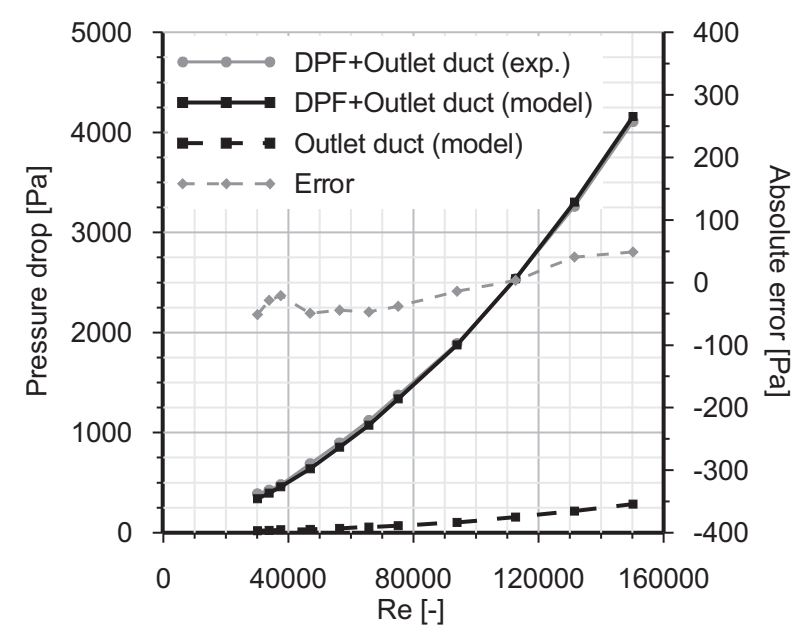

Figure 5: Comparison between measured and modelled pressure drop across the DPF and the outlet duct when the iterative characterisation procedure is converged.

a)

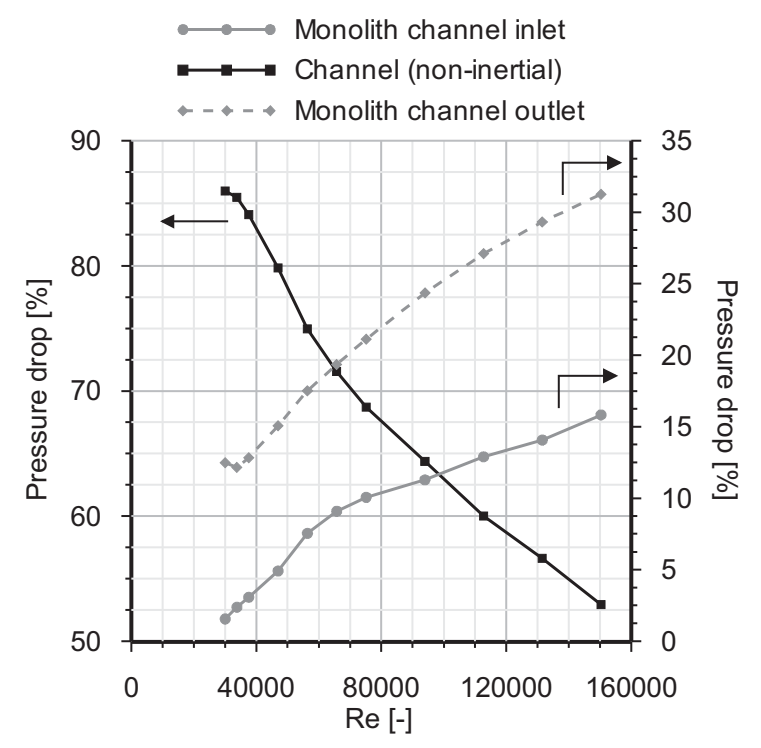

b) $\quad \bullet \rightarrow-\bullet$ Inlet cone

$\longrightarrow$ Monolith channel inlet

$\longrightarrow$ Channel (non-inertial)

$\rightarrow-\rightarrow$ Monolith channel outlet

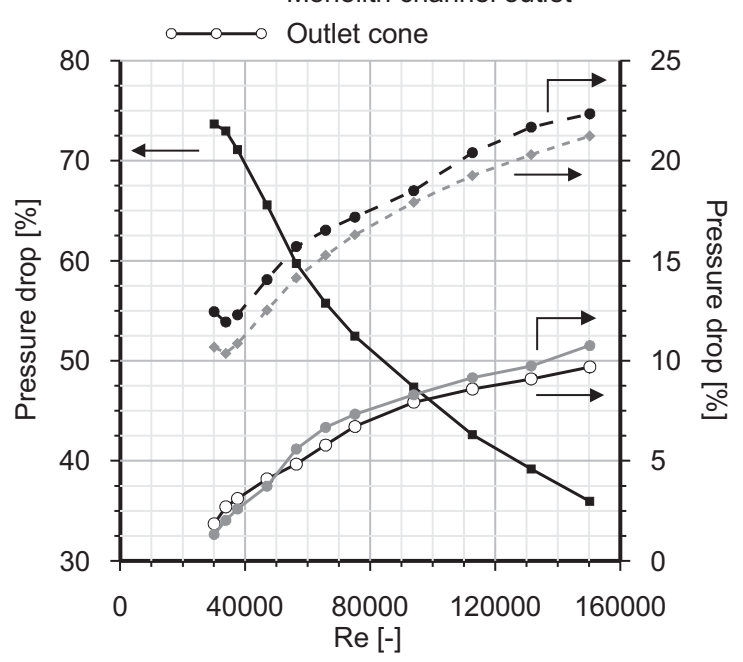

Figure 6: Decomposition of the pressure drop contributions: a) Monolith; b) canned DPF. 


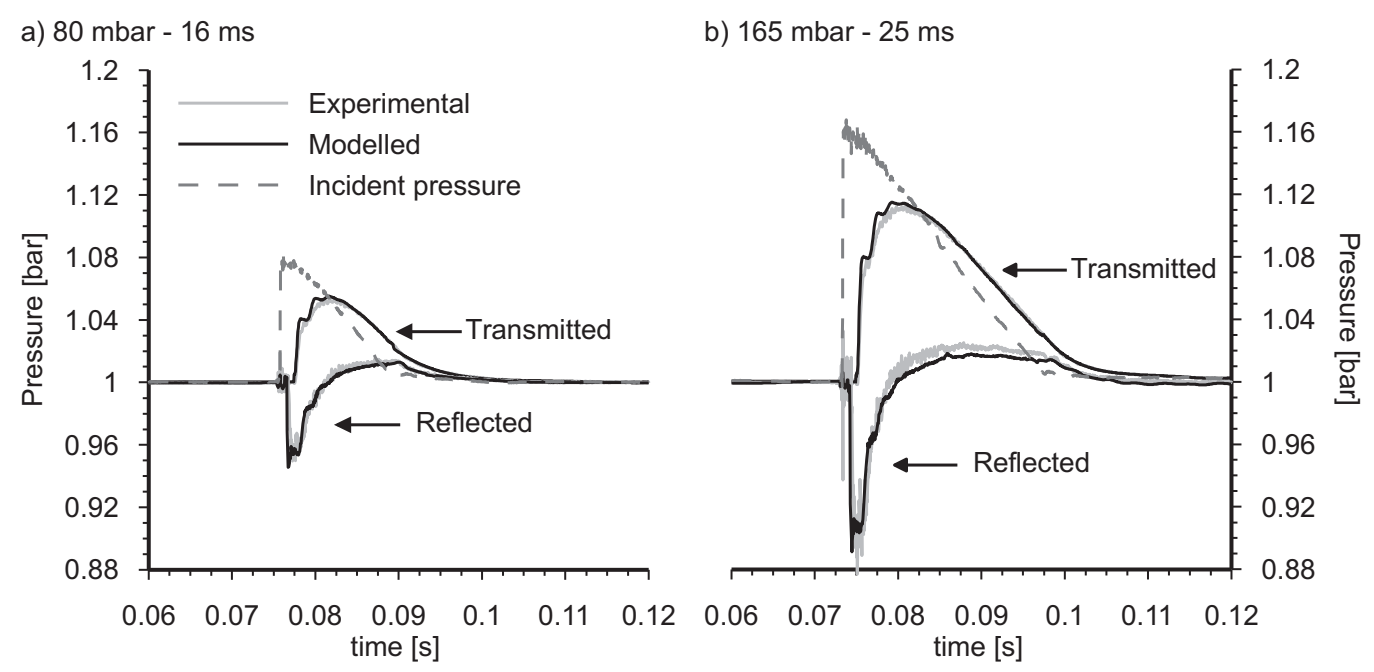

Figure 7: Experimental and modelled results in time domain under impulsive flow conditions.

a) $95 \mathrm{mbar}-11 \mathrm{~ms}$

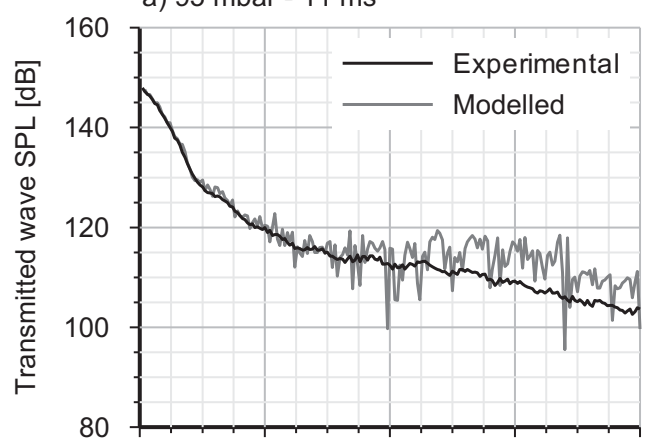

b) $200 \mathrm{mbar}-20 \mathrm{~ms}$

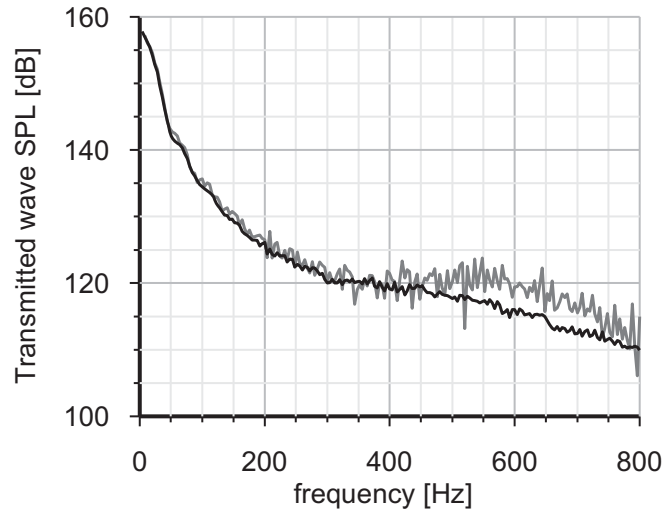

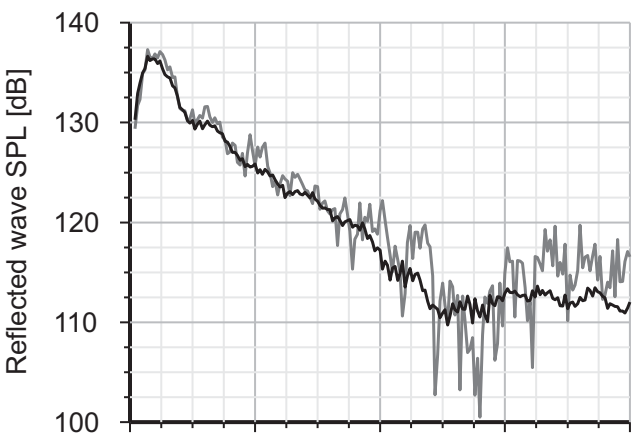

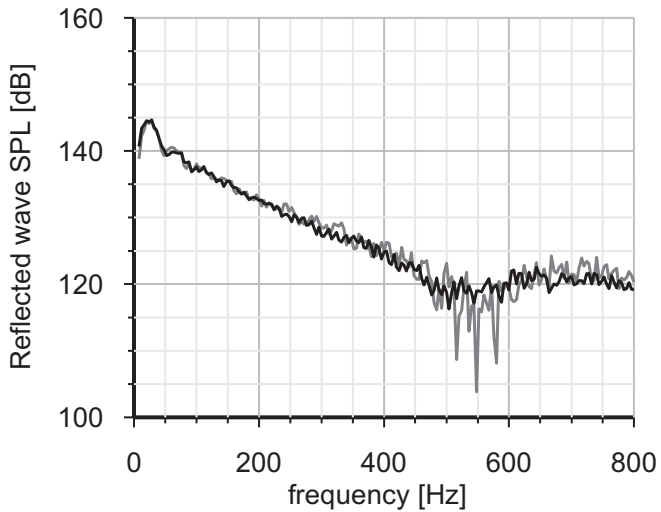

Figure 8: Experimental and modelled results in frequency domain under impulsive flow conditions. 


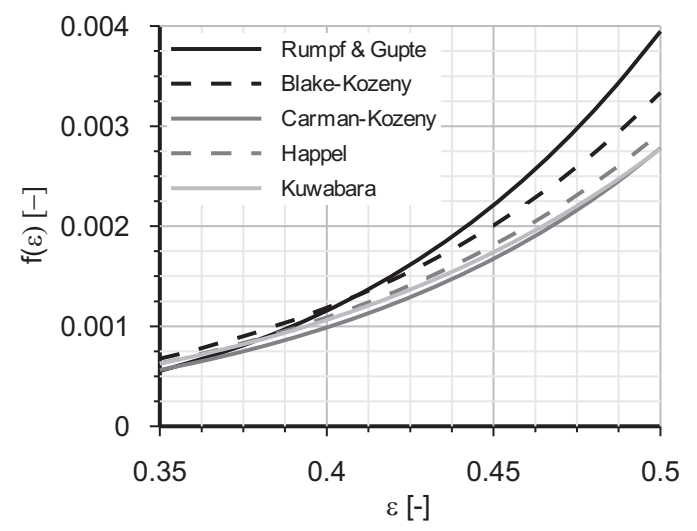

Figure 9: Porosity factor value for different flow models in packed beds of spherical particles.

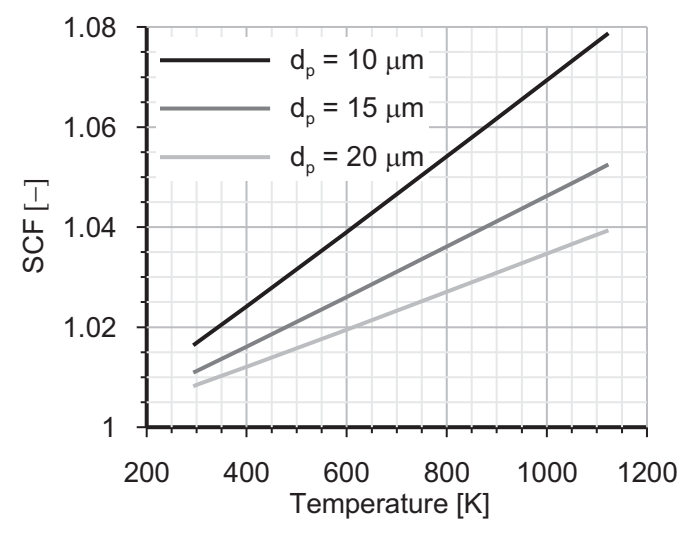

Figure 10: Stokes-Cunningham factor for slip-flow correction of the continuum permeability.

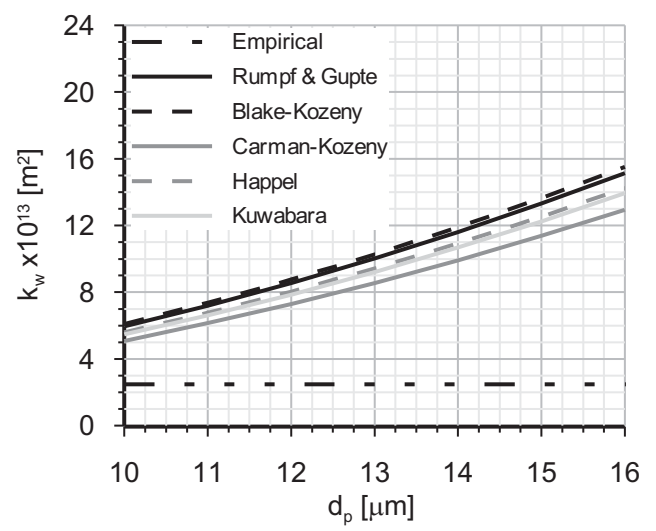

Figure 11: Disagreement between empirical and calculated permeability as a function of several porosity factor correlations $(\varepsilon=0.4)$. 
a) Kuwabara (Procedure 1)

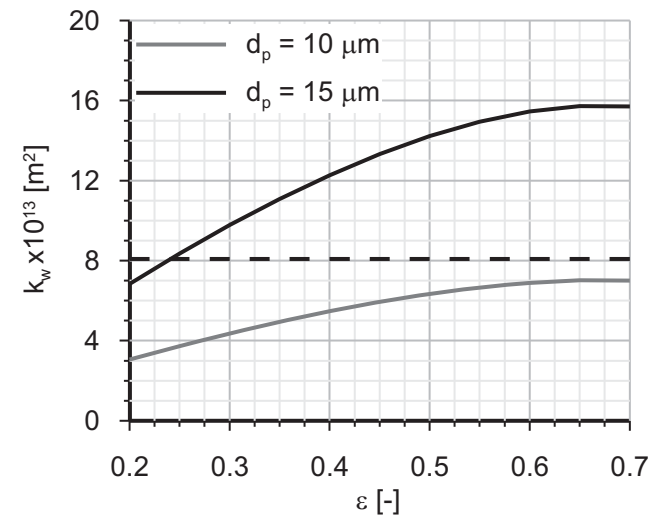

b) Kuwabara (Procedure 2)

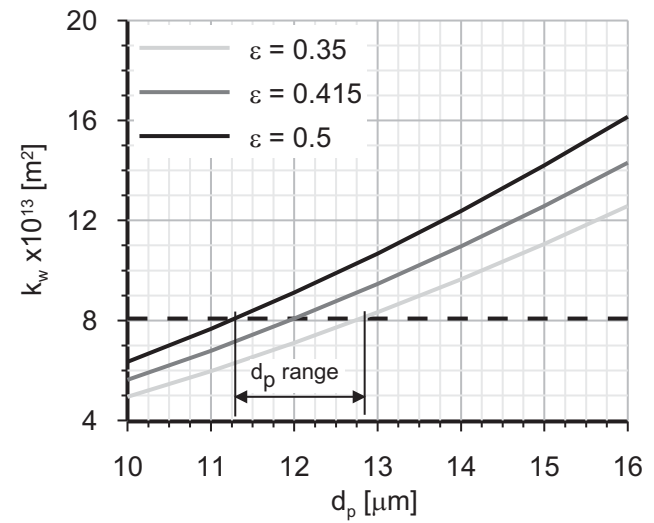

Figure 12: Procedures for porosity and mean pore diameter determination from continuum permeability and Kuwabara's porosity factor.

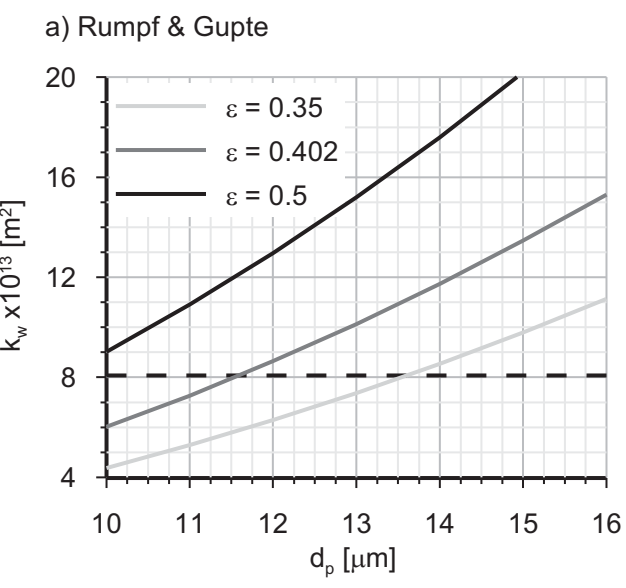

c) Carman-Kozeny

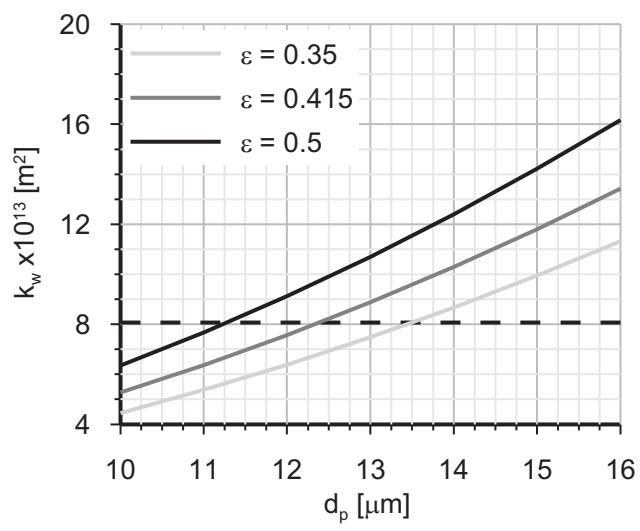

b) Blake-Kozeny

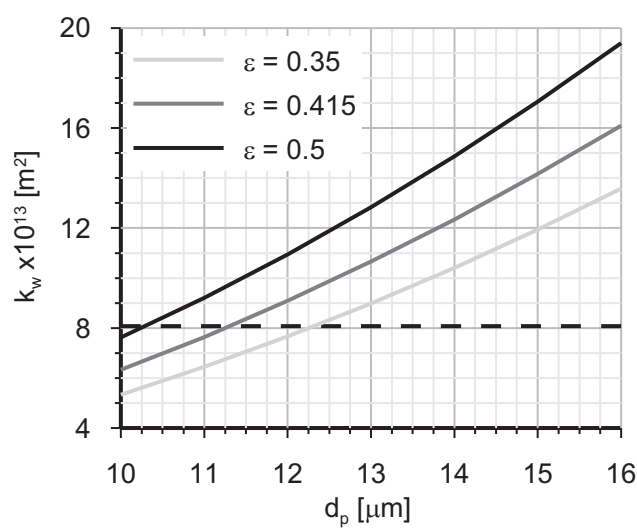

d) Happel

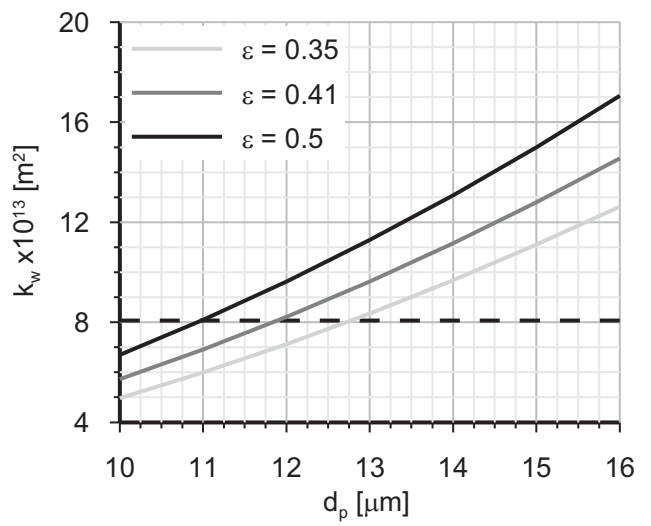

Figure 13: Permeability as a function of $d_{p}$ and $\varepsilon$ for several porosity factor models. 


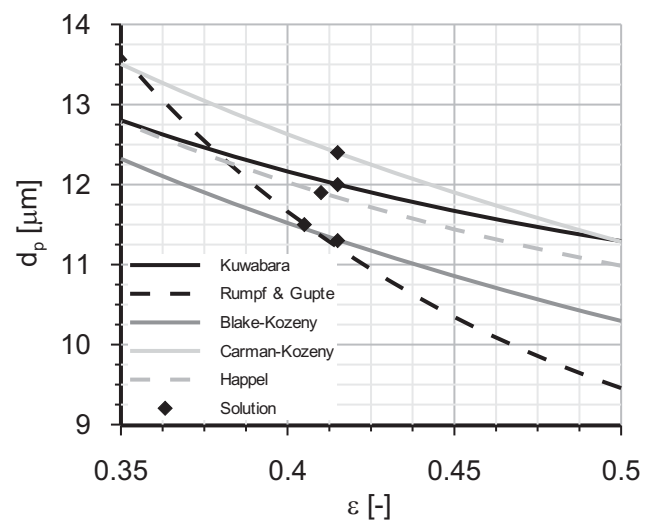

Figure 14: Figure 14. Iso-lines of $k_{w}=8.076 \cdot 10^{-13} \mathrm{~m}^{2}$ for every porosity factor model and solution provided applying Procedure 2.
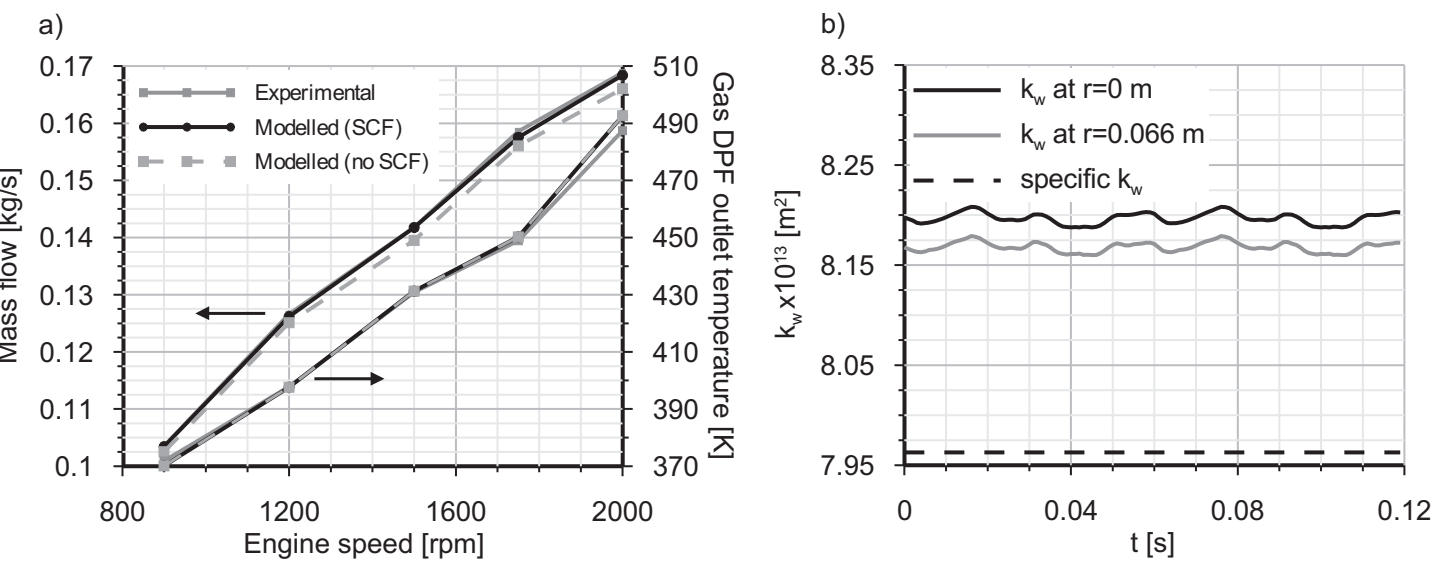

Figure 15: DPF response under hot pulsating flow conditions. a) Comparison between measured and predicted mass flow and downstream DPF temperature by the 1D wall-flow DPF model. b) Continuum permeability at two monolith radial locations at $2000 \mathrm{rpm}$ and comparison against specific permeability of the porous wall.
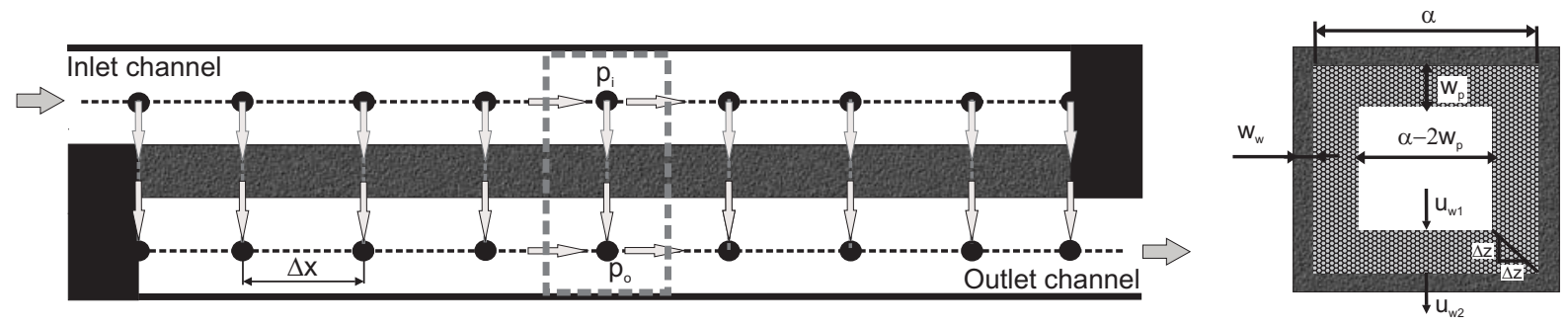

Figure 16: Axial and cross-section geometry of channels in wall-flow DPF monoliths and description of the spatial mesh and filtration velocity through the porous medium. 